\title{
Research Trends in Holographic 3DTV Displays
}

\author{
Levent Onural \\ Dept. of Electrical and Electronics Eng. \\ Bilkent University \\ TR-06800 Ankara, Turkey \\ 1.onural@ieee.org
}

\begin{abstract}
Holographic 3DTV and especially displays are attracting more attention. There are SLM based laboratory prototypes. Current images can be viewed from a very narrow angle. Satisfactory holographic displays can be expected within a decade.
\end{abstract}

\section{BRIEF HISTORY}

Interest in 3D video is rising [1]. All functional units in the $3 \mathrm{D}$ video production and delivery chain, like capture, transmission and display are important for a good quality, but the display has a special significance since the end-user interacts directly with it [2], [3]. However, most of the 3D video systems in the past, and at present, are based on stereoscopy. Stereoscopy is based primarily on human perception and has many problems; a well known deficiency of stereoscopy reveals itself as "eye fatigue" which is a term used to describe the motion sickness type of a feeling that the viewers get within minutes of viewing experience, especially if the stereoscopic video has alignment problems. Perfect alignment, usually achieved as a consequence of end-to-end digital technologies, reduces the eye fatigue, but intrinsic focus-vergence mismatch still creates an unusual viewing experience. True $3 \mathrm{D}$, on the other hand, is a term which describes viewer independent physical duplication of volume-filing light fields. A true 3D system attempts to record light distributions, and then tries to create exact optical duplicates of the original light distribution, at another place, and maybe at another time. If the duplication is perfect, any observer, human or else, who is interacting with the recreated light will see exactly the same 3D environment as if looking into the original 3D scene, since the recreated light field will be the same as the original. Holography is a true 3D imaging technique. However, many parameters and physical restrictions associated with holography still prevent perfect duplication, and thus fidelity degrades. Integral imaging can also be categorized as a form of true 3D imaging technique provided that large microlens arrays are employed. Successful true 3D imaging systems, and therefore, displays are essential in achieving ghost-like images as depicted in many popular movies.

It is absolutely necessary to record the intensity and direction of light as it propagates in a 3D volume for a successful $3 \mathrm{D}$ recording. Any recording device, including classical video cameras, somehow record some degree of directional information in addition to intensity. However, a classical 2D camera has a very restricted directional recording ability, and that is far from being adequate for a satisfactory 3D replay. Better quality $3 \mathrm{D}$ replay is achieved as degree of directional recording ability increases. Directional (and intensity) distribution is recorded in the form of interference patterns in holography.

Still holography has been demonstrated since 1960's [4]. Holographic movies are also made. Electro-holography is a term used since 1980's to describe electronically re-writable holographic display devices so that a holographic video (holographic 3DTV) can be achieved. An early electro-holography display device that used acousto-optic modulators was Mark I of MIT [5]. More recent systems are based on liquid crystal devices, focused light arrays, optically addressed spatial modulators or digital micromirror devices [3]. However, holographic video displays are still in their infancy: there are only a few laboratory systems with quite limited capabilities. Reduced forms of holographic displays, such as eye-tracking single viewer devices, have been recently commercialized [6].

\section{An OVERVIEW of Holographic Video Displays}

Almost all holographic displays are currently flat-surface devices with small sizes. It is desirable to write a complex valued interference pattern on such a device which will then diffract the light. Transmissive systems diffract the light passing through them, whereas, reflective devices operate in a mirror-like manner by diffracting the reflected light. The diffraction angle is a function of $2 \mathrm{D}$ pattern frequency over the device, where large diffraction angles are possible if high spatial frequency diffraction patterns can be written. Therefore, it is highly desirable to have display devices which can support as high as possible spatial frequency patterns. Since most of the currently available devices are pixellated arrays, this means that very small pixel sizes are needed; pixels whose sizes are in the order of wavelength are highly desirable. Liquid crystal (LC) spatial light modulators (SLMs) are commonly used for such purposes. Liquid crystal on silicon (LCoS) SLMs are quite popular [3], [7]. However, currently available minimum pixel size is in the order of a few microns (typical pixel sizes are 6-20 $\mu \mathrm{m}$ ). Unfortunately, only a few degrees of diffraction angles are possible with such rather large pixel size devices. Furthermore, currently available SLM sizes are in the full-HDTV range with approximately $2 \mathrm{~K}$ by $1 \mathrm{~K}$ pixel arrays. The physical size is in the order of $2 \mathrm{~cm} \times 1 \mathrm{~cm}$. That leads to quite small reconstructed images which can only be seen from a very narrow viewing angle. Another 
problem associated with a pixellated device is the higher order diffractions that result in many undesirable duplicates of the reconstruction. Therefore, it is highly desirable to have a large fill-factor, and a smooth surface across the entire SLM to reduce the power in higher diffraction orders. Yet another common problem is the difficulty in writing complex valued patterns; almost all practical systems are either magnitudeonly, or phase-only devices. Losing either the magnitude or phase variation degrades the reconstruction quality; phase-only systems provide better performance compared to magnitudeonly systems in terms of quality.

A holographic display device which dynamically writes holographic fringes on a surface acoustic wave crystal by feeding multiple time-varying signals was also proposed [8].

It is also possible to use conventional LCD panels that are intended for computer or TV monitors as holographic displays. However, very large pixel sizes will result in extremely small diffraction angles. A commercial system uses such a display, but then employes a directionally adjustable oblique illumination to yield the larger angles [6].

Coherent illumination (lasers) are inevitable for holographic recording. However, holographic display devices does not necessitate holographic recordings to operate. An alternative is to capture $3 \mathrm{D}$ scene information by non-holographic means, and then compute holograms for the displays using appropriate algorithms [9]. Efficient computational procedures are needed for real-time video operation with refresh rates that are in the order of many tens of frames per second for video operation [10], [11]. Such computational algorithms are based on signal processing techniques, and many variants have emerged [12]. Fortunately, coherence requirements during the display are much less stringent. Even narrow-band, spatially filtered (pin-hole) LED illumination is demonstrated to be adequate. Multiple SLMs are used for color reconstructions [13], [14].

Current research is concentrating on novel efficient algorithm development using sophisticated signal processing techniques, novel SLM devices with smaller pixel sizes and larger arrays, better SLMs with properties like linear and large dynamic range, novel rewritable polymer devices based on nanotechnology to support large interference patterns with high-resolution, and novel designs based on multiple SLMs and time-multiplex methods.

\section{CONCLUSION}

Research in holographic video displays are gaining more and more momentum. Currently, there are experimental an limited-capability holographic display devices. However, emerging techniques are expected to advance the state-of-the art quite quickly. It will not be surprising to see successful holographic displays which can support reasonable size ghostlike 3D reconstructions within a decade.

\section{ACKNOWLEDGMENT}

This work is supported by European Community within FP7 under the grant agreement 216105 with an acronym Real 3D.

\section{REFERENCES}

[1] L. Onural, "Television in 3D: What are the prospects?" Proceedings of the IEEE, vol. 95, no. 6, pp. 1143-1145, Jun 2007.

[2] H. M. Ozaktas and L. Onural, Eds., Three-Dimensional Television: Capture, Transmission and Display. Springer, 2008.

[3] P. Benzie, J. Watson, P. Surman, I. Rakkolainen, K. Hopf, H. Urey, V. Sainov, and C. von Kopylow, "A survey of 3DTV displays: Techniques and technologies," Circuits and Systems for Video Technology, IEEE Transactions on, vol. 17, no. 11, pp. 1647-1658, Nov. 2007.

[4] E. N. Leith and J. Upatnieks, "Reconstructed wavefronts and communication theory," JOSA, vol. 52, pp. 1123-1130, 1962.

[5] J. S. Kollin, S. A. Benton, and M. L. Jepsen, "Real-time display of 3-D computed holograms by scanning the image of an acoustooptic modulator," in Proc. SPIE, Holographic Optics II: Principles and Applications, vol. 1136, 1989, pp. 178-185.

[6] S. Reichelt, R. Haussler, N. Leister, G. Futterer, and A. Schwerdtner, "Large holographic 3D displays for tomorrow's TV and monitors solutions, challenges, and prospects," IEEE Lasers and Electro-Optics Society, 2008. LEOS 2008. 21st Annual Meeting of the, pp. 194-195, 2008.

[7] M. Kovachev, R. Ilieva, P. Benzie, G. B. Esmer, L. Onural, J. Watson, and T. Reyhan, "Holographic displays using spatial light modulators," in Three-Dimensional Television: Capture, Transmission, Display, H. M. Ozaktas and L. Onural, Eds. Springer, 2008.

[8] L. Onural, G. Bozdağı , and A. Atalar, "New high-resolution display device for holographic three-dimensional video: Principles and simulations," Opt Eng, vol. 33, pp. 835-844, 1994.

[9] M. Janda, I. Hanak, and L. Onural, "Hologram synthesis for photorealistic reconstruction," Journal of the Optical Society of America A, vol. 25, no. 12, pp. 3083-3096, December 2008.

[10] H. Kang, F. Yaras, L. Onural, and H. Yoshikawa, "Real-time fringe pattern generation with high quality," in Digital Holography and ThreeDimensional Imaging Conference, OSA, April 2009.

[11] H. Kang, F. Yaras, and L. Onural, "Quality comparison and acceleration for digital hologram generation method based on segmentation," in Proc. of 3DTV-CON 2009, IEEE, May 2009.

[12] L. Onural, A. Gotchev, H. M. Ozaktas, and E. Stoykova, "A survey of signal processing problems and tools in holographic three-dimensional television," IEEE Tr. on Circuits and Systems for Video Technology, vol. 17, no. 11, pp. 1631-1646, November 2007.

[13] F. Yaras, H. Kang, and L. Onural, "Real-time multiple slm color holographic display using multiple gpu acceleration," in Digital Holography and Three-Dimensional Imaging Conference, OSA, April 2009.

[14] F. Yaras and L. Onural, "Color holographic reconstruction using multiple slms and led illumination," in Proc. SPIE, vol. 7237, January 2009. 